\title{
Stochastic programming to evaluate renewable power generation for small-scale desalination
}

\author{
R. B. Webby ${ }^{1} \quad$ J. Boland ${ }^{2} \quad$ A. V. Metcalfe $^{3}$
}

(Received 26 July 2007; revised 8 November 2007)

\begin{abstract}
Due in part to an increasing population and climatic change, fresh water demand is rapidly outpacing fresh water supply. In Australia desalination plants are already used to obtain fresh water from brackish water and seawater, but they have high energy requirements. Solar collectors could provide power, but solar irradiance is variable and desalination plants work most efficiently with constant power. We model a system of photovoltaic arrays and storage batteries. Daily solar intensity and water demand are stochastic. A stochastic linear program finds the optimal blend of water from available sources - groundwater, desalination and stormwater - to meet daily demand. The optimal use of a given size of solar irradiance collection system is found by stochastic dynamic programming. Long term net benefits are obtained as a function of the system size.
\end{abstract}

See http://anziamj.austms.org.au/ojs/index.php/ANZIAMJ/article/view/320 for this article, (c) Austral. Mathematical Soc. 2007. Published November 14, 2007. ISSN $1446-8735$ 


\section{Contents}

1 Introduction

C185

2 Stochastic dynamic program $\quad$ C186

2.1 Defining the state space . . . . . . . . . . . . C C187

2.2 Solar irradiance input to the system . . . . . . . . . . . C C191

2.3 Optimal policies . . . . . . . . . . . . . . . . C191

3 Stochastic linear program

C194

4 Conclusions

C196

References

C197

\section{Introduction}

People living in arid and semi-arid Australia frequently face a shortage of potable water. But remote communities, national parks, stations and islands often have access to saline groundwater or sea water, along with abundant solar energy. These locations are generally not connected to the electricity grid and researchers are investigating autonomous systems of desalination modules powered by renewable energy for such locations. Investigations include pilot projects in Australia [6] and the Mediterranean region [1], and mathematical models of renewable powered desalination plants to simulate their operation $[4,5]$. Both practical and mathematical models aim to provide guidance for system sizing and efficient operational strategies. The potentially useful technique of stochastic programming has been used to analyse both short term and long term planning issues in the allied arena of the deregulated electricity market [3]. However, it has not been used in decision making for renewable power generation for small scale desalination systems. 
We describe a mathematical model of a solar energy powered, reverse osmosis desalination plant using stochastic programming to assess system sizing, operating rules and longer term objectives of water production. The plant comprises a photovoltaic (PV) array connected to an energy storage system of batteries and to two desalination modules that can be run independently of each other. A stochastic dynamic programming (SDP) algorithm finds the optimal policy for the allocation of energy flows from the PV array while meeting typical plant operating conditions. Results from the SDP are an input to a stochastic linear program (SLP) which matches water demand to supply at lowest cost while meeting availability and salinity constraints.

\section{Stochastic dynamic program}

The SDP algorithm uses transition matrices whose entries, $p_{i j}(k)$, which depend on a decision $k$, are the probability of moving between states of the state space, and a reward matrix whose entries, $r_{i j}(k)$, are the value obtained by making a particular transition under decision $k$. Let $i \in\{1,2, \ldots, m\}$ represent the states of the system. For our model, $m=18$. Let $t \in\{0,1, \ldots, T\}$ be discrete one hour time periods. The policy iteration procedure is implemented in two parts: value determination and policy improvement [2]. For a given policy, total expected earnings over the remaining time steps at time $t$ depends on the state, $i$, at time $t$, and is written $v_{t}(i)$. For a given policy, total expected earnings is calculated recursively as

$$
v_{t}(i)=\sum_{\text {all states } j} p_{i j}\left[r_{i j}+v_{t+1}(j)\right], \quad \text { for } j=1, \ldots, m .
$$

For large $t$,

$$
v_{t}(i)=g+v_{t+1}(i)
$$


where $g$ is the expected return per period. Substituting into (1) gives the set of equations making up the value determination step:

$$
g+v(i)=\sum_{j} p_{i j} r_{i j}+\sum_{j} p_{i j} v(j), \quad i=1, \ldots, m .
$$

These equations are solved for $g$ and $v(2)$ up to $v(m)$ with $v(1)$ being arbitrarily set to 0 in order to obtain a solution for the under determined system. The policy improvement step maximises for all states $i$

$$
\sum_{j} p_{i j}^{\prime}(k) r_{i j}^{\prime}(k)+\sum_{j} p_{i j}^{\prime}(k) v(j) .
$$

The algorithm starts with an arbitrary policy and continues until the policies produced on two successive iterations are identical.

\section{Model assumptions}

We assume that the process of desalination requires a desalination module to be run for two hours to produce a unit quantity of desalinated water, and that a module run for one hour produces no potable water. This assumption recognises that for efficient operation of reverse osmosis modules, the water pressure and the brine to feedwater ratio in the modules must be carefully regulated to ensure the quality of the water produced, to manage and dispose of the brine stream, and to minimise scaling of membrane surfaces. We assume that a module uses one unit of energy per hour when running.

\subsection{Defining the state space}

The time step of the model is one hour, a period of similar scale to the desalination process, avoiding excessive start/stop operations but allowing the system to take advantage of favourable conditions. The time scale could 
be altered if there is evidence that this is necessary. We specify discrete states for each desalination module of $\{0,1,2\}$, where state ' 0 ' represents the module being unused, state ' 1 ' represents the module having completed the first hour of the desalination process, and state '2' represents the module having completed the second hour of the desalination process. We specify discrete states for the storage level of the battery assemblage and assume the assemblage has a storage capacity of $B$, so that $b \in\{0, B\}$ represents the number of recoverable units of energy held in storage. For the initial formulation of the problem we set the states of the battery assemblage to be $\{0,1\}$. Thus storage capacity is one unit and we assume that excess energy cannot be used in this application.

The state space of the problem is made up of triplets, $\left(m_{1} m_{2} b\right)$, where $m_{1} \in\{0,1,2\}$ records the state of module $1, m_{2} \in\{0,1,2\}$ records the state of module 2 , and $b \in\{0,1\}$ records the state of the battery storage. There are three possible states for each desalination module and two for the battery assemblage, giving 18 combinations. The state space of the problem, in the order arbitrarily chosen here, is

$$
\begin{aligned}
& \{(000),(010),(020),(001),(011),(021),(100),(110),(120), \\
& (101),(111),(121),(200),(210),(220),(201),(211),(221)\} .
\end{aligned}
$$

We consider three decisions, $k \in\{0,1,2\}$ (Figure 1): 0 , run no desalination modules; 1 , run one desalination module only; 2 , run both desalination modules.

A decision is made at hourly intervals at the beginning of a time period in the knowledge of the state of the system and the probability of energy inflows for the next hour. The decision is made for the time period immediately following and energy flows during that time period are directed according to the decision.

Energy inflows from the PV array are stochastic and we model inflow amounts to be compatible with the discrete quantities of the state space. 
Photovoltaic array

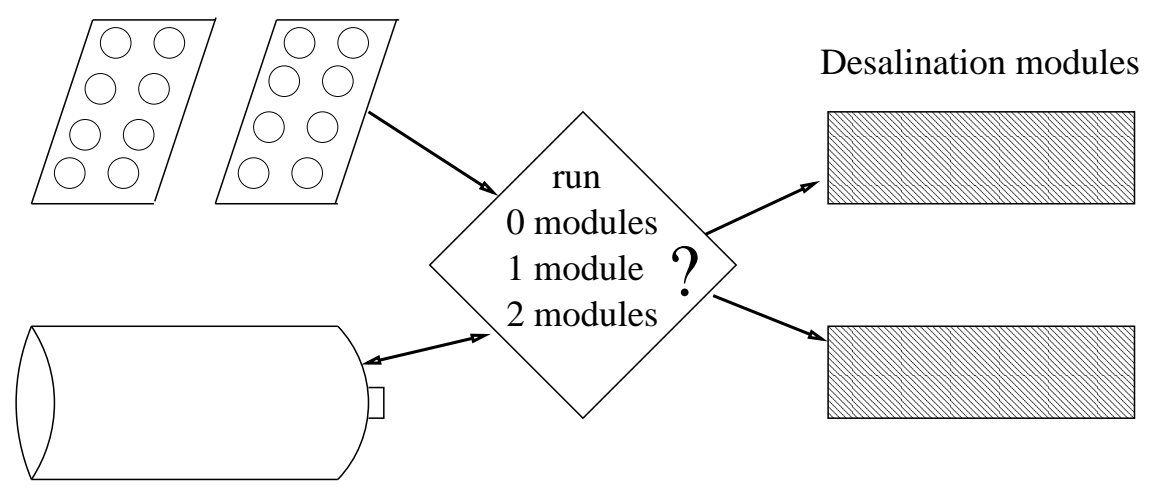

Energy storage

Figure 1: The process control problem

Thus, the PV array will supply 0,1 or 2 units of energy in an hour with probabilities $p_{0}, p_{1}$ and $p_{2}$ respectively. Possible transitions between states depend on the decision made and on the following conventions for energy use which aim to reproduce likely operational procedures.

- Energy from the PV array is first directed to any desalination module that is running and then to the storage system. Excess energy cannot be used in this application.

- If there is a choice between using a unit of energy for running one desalination module for a first hour or directing that energy to running one desalination module for a second hour, then the latter action is taken.

- If one module has been idle while the other has just completed a two hour run and a decision is taken to run one module, then the idle module is selected.

- If both modules have just completed a one hour run or a two hour run and a decision is taken to run one module, then the module represented 
by the first element of the state space triplet is selected.

We illustrate the calculation of the entries of the transition matrices. Decision 2 is to run two desalination modules. In state (000), for example, neither module has been in use in this time step and no energy was stored. A decision is taken to run two modules. With probability $p_{0}$, no energy is available from the PV array during the current time step, the desalination process is halted and the system remains in state (000). With probability $p_{1}$, one unit of energy is obtained through the PV collectors and the system moves from state (000) to state (100). With probability $p_{2}$, the system moves to state (110).

A cost of $r$ is incurred when a desalination module is run for up to one hour. The cost includes pretreatment of the feedwater such as screening and filtering, chemical treatment of cations and storage of cleaned feedwater. The cost also includes backflushing and eventual replacement of membranes, disposal of brine and storage of the product. A benefit of $r$ is assigned for completing the first hour of desalination. Thus a transition to state (100), say, has a reward of $-r+r=0$. A benefit of $2 r$ is obtained for completing the second hour of desalination. Thus an eventual transition to state (200), say, has a reward of $-r+2 r=r$. Any decision taken is implemented at the beginning of a time step. If a decision is taken to run a module but there is insufficient energy to complete the run, the cost of running the module is incurred without any benefit, thus the reward is $-r$ per module started. The net result of this is that an overall benefit of $r$ accrues if a desalination module completes two hours of running and an overall loss (benefits minus costs) of $r$ accrues if the module is run and fails to complete one hour or fails to complete two hours. 


\subsection{Solar irradiance input to the system}

We characterise energy levels in the system as being of 0,1 or 2 units where an energy level of 0 units is insufficient to run a desalination module for one hour, a level of 1 unit is sufficient energy to run one desalination module for one hour, and a level of 2 units is sufficient to run one desalination module for two hours. We use a 38 year data record to characterise energy input to the PV array and model solar irradiance for January and July - potentially the months of greatest and least solar energy. Average hourly direct beam solar insolation for Adelaide for the period from 6 am to $6 \mathrm{pm}$ in January and $8 \mathrm{am}$ to $4 \mathrm{pm}$ in July has a similar distribution of intensity for each hour, and so we aggregated the data to represent a typical hour's insolation for these two periods (Figure 2). We set the ranges of solar irradiance that constitute 0,1 or 2 units of energy as: 0 units for solar irradiance between 0 and $150 \mathrm{Whm}^{-2}$; 1 unit for solar irradiance between 150 and $450 \mathrm{Whm}^{-2}$; and 2 units for solar irradiance between 450 and $1150 \mathrm{Whm}^{-2}$. Thus the probabilities of irradiance amounts within the three ranges of direct beam solar irradiance falling on a dual-tracking PV array are, for January 6 am to $6 \mathrm{pm}, 0.15,0.21$ and 0.64 respectively, and, for July 8 am to $4 \mathrm{pm}, 0.37$, 0.30 and 0.33 respectively. We assume that the energy produced by the PV array is a linear function of solar irradiance but a more detailed model would include the degradation of array performance at higher temperatures [5].

\subsection{Optimal policies}

A policy specifies a decision for each state of the system. We write a policy as a vector with the elements of the vector representing the states of the system in our chosen order and the entries of the vector as the respective decisions. 

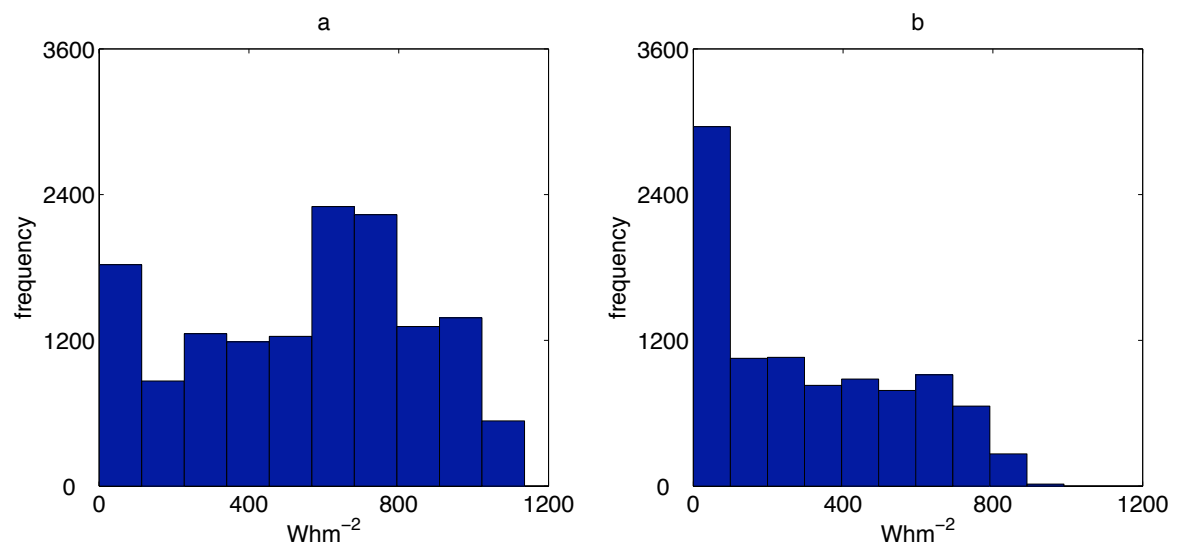

FiguRE 2: Direct beam solar insolation at Adelaide for daylight periods: (a) January, 6 am to $6 \mathrm{pm}$; and (b) July, 8 am to $4 \mathrm{pm}$.

\section{July, 8 am-4 pm}

SDP analysis gives an optimal policy of

$$
\text { [0 } 101011111112101011 \text { ] . }
$$

There is one state in which it is optimal to run both modules simultaneously, but, under the optimal policy, the system cannot reach this state. In summary, the operating rules under this policy are: if a desalination module has just completed the first hour of the process, run it for the second hour; if both modules are available and there is energy in storage, start one module; else, run no modules. Taking this latter decision means, in practical terms, that any incoming energy is used to build up stored energy.

The average, long term, desalinated water production under this policy is 0.31 units per hour. Note that either one or no module is run in any time step and thus the plant is under utilised. 


\section{January, 6 am-6 pm}

SDP analysis gives an optimal policy of

$$
\text { [1 } 111221121222111121] \text {. }
$$

The operating rules under this policy are: run at least one desalination module in any state; run two modules if one of the modules has just completed its first hour of desalination and the system has stored energy, and if both modules have just completed their first hour of desalination but with no energy in storage.

The average, long term, desalinated water production under this policy is 0.70 units per hour. At least one desalination module is run at each time step and thus the system is utilised more fully under this policy. The hourly desalinated water production in July is approximately $44 \%$ of hourly production in January. However, due to the longer period of daylight and thus the extended operational time in January, daily desalinated water production in July is approximately $30 \%$ that of January.

\section{Expanded storage states}

During the design phase of a photovoltaic system particular attention is given to deciding on the relative sizes of the solar collector array and an energy storage system. For example, sufficient storage capacity may be provided to run a plant at its average production rate for one to two days without external energy input. As an extension to our basic model, we expand the state space by doubling potential storage capacity, so that the states of stored energy are $\{0,1,2\}$. The state space for the problem is now

$$
\begin{aligned}
& \{(000),(010),(020),(001),(011),(021),(002),(012),(022), \\
& (100),(110),(120),(101),(111),(121),(102),(112),(122),
\end{aligned}
$$




$$
\text { (200), (210), (220), (201), (211), (221), (202), (212), (222)\}. }
$$

Rewriting transition and reward matrices to follow model assumptions and conditions as before and conducting SDP analysis gives an optimal policy for July, 8 am to $4 \mathrm{pm}$ of

$$
\left[\begin{array}{llllllllllllllllllllllll}
0 & 0 & 0 & 1 & 1 & 1 & 1 & 0 & 0 & 0 & 1 & 1 & 2 & 2 & 0 & 0 & 0 & 1 & 1 & 1 & 1 & 2 & 1
\end{array}\right],
$$

and an optimal policy for January, 6 am to $6 \mathrm{pm}$ is

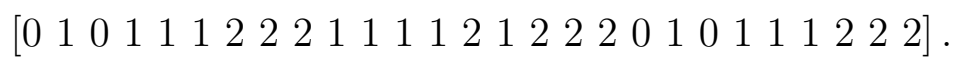

The average, long term, desalinated water production for July is 0.38 units per hour while that for January is 0.72 units per hour. Thus extra energy storage increased water production by $23 \%$ during July and by $3 \%$ in January. Thus, system sizing is adequate for January but added storage capacity could increase water production during July. Note that water demand may be lower in July.

\section{Stochastic linear program}

We consider daily household and agricultural demands for water for a small community in January. Household use includes cooking, drinking and washing while agricultural use includes drinking water for stock and irrigation of food crops. We consider three sources of supply of water: rainfall as stochastic; a low salinity source of groundwater as deterministic; and output from the desalination plant also as stochastic. The triangular distribution for desalinated water production is suitable for such a process where the maximum, minimum and most likely values are known. The gamma $(49,7)$ distribution produces random rainfall that satisfies, on average, one third of demand. Demand is modelled as a bivariate normal distribution for household and 
TABLE 1: Water characteristics of sources and sinks

\begin{tabular}{lccc}
\hline source & rain & ground & desal \\
\hline availability (units) & $\sim$ gamma $(49,7)$ & up to 9 & $\sim \operatorname{triang}(0,12)$ \\
salinity $(\mathrm{mg} / \mathrm{l})$ & 100 & 1500 & 500 \\
\hline \hline sink & & household & agricultural \\
\hline demand (units) $($ correlation $=0.6)$ & $\sim \mathrm{N}\left(1.5,0.2^{2}\right)$ & $\sim \mathrm{N}\left(15,1.2^{2}\right)$ \\
maximum salinity $(\mathrm{mg} / \mathrm{l})$ & 500 & 900 \\
\hline
\end{tabular}

agricultural uses with average agricultural demand ten times that of household demand. These are positively correlated to reflect similar patterns of demand from both uses during similar climatic conditions (Table 1).

Let $r, g$ and $d$ represent the sources of rain, ground and desalinated water, respectively. Let $h$ and $a$ represent the demand sites of household and agricultural use. Let $x_{i j}$ be the amount of water supplied from source $i$ to demand site $j$ and $c_{i j}$ be the cost of such supply. We write the quantity of water available from source $i$ in a given time step as avail $i$, the demand at site $j$ as $\operatorname{dem}_{j}$, and the salinity conditions of the sources and demand sites as $\mathrm{sal}_{i}$ and $\mathrm{sal}_{j}$ respectively. The linear program is

$$
\begin{aligned}
\min & \sum_{i j} c_{i j} x_{i j}, \\
\text { such that } \quad & \sum_{i} x_{i j} \geq \operatorname{dem}_{j} \quad \text { for } j=h, a \\
& \sum_{j} x_{i j} \leq \operatorname{avail}_{i} \quad \text { for } i=r, g, d, \\
& \left(\sum_{i} \operatorname{sal}_{i} x_{i j}\right) /\left(\sum_{i} x_{i j}\right) \leq \operatorname{sal}_{j} \text { for } j=h, a \\
& x_{i j} \geq 0 .
\end{aligned}
$$

The program is run multiple times to simulate demand and supply for the application, with the algorithm sampling from probability distributions each 
TABLE 2: Percentage use of source rain, ground, desal for varied cost of desalinated water

\begin{tabular}{lrrr}
\hline cost structure & $r$ & $g$ & $d$ \\
\hline $1,0.5,5$ & 90 & 100 & 15 \\
$1,0.5,2.5$ & 86 & 100 & 30 \\
$1,0.5,1$ & 53 & 100 & 40 \\
\hline
\end{tabular}

time to generate values for the stochastic variables. Results in Table 2 show that use of desalinated water is price sensitive as would be expected. For the three scenarios of cost structure, supply fails to meet salinity conditions on approximately $3 \%$ of occasions. Desalinated water supplies are not fully used even when priced equal to rainwater.

If we suppose mean rainfall supplies only one quarter of January demand, then use of desalinated water increases. For a cost schedule of $1,0.5,5$ approximately $57 \%$ of desalinated water is used, compared to $100 \%$ and $90 \%$ of ground and rain water. However this scenario also sees an increase to approximately $11 \%$ in the frequency of failure to supply water of acceptable quality - a rate that may be unacceptable.

\section{Conclusions}

Stochastic dynamic programming determines efficient operating strategies for the use of energy inflows for an autonomous, solar energy powered, reverse osmosis desalination system. The analysis evaluated system sizing and calculated expected water production. Results from the SDP were input to a stochastic linear program which assessed the contribution desalinated water might make to meeting demand in a small community. It could be worthwhile extending this analytic approach of assessing an intended installation using actual data. An improvement in the model would be to couple its two 
parts so that the percentage use of desalinated water in the SLP influences the reward for producing water in the SDP.

Acknowledgements: We thank the ARC for supporting this research under grant number DP0559399. We thank David Green of the University of Adelaide for providing Matlab code for the stochastic dynamic program.

\section{References}

[1] H. E. S. Fath, F. M. El-Shall, G. Vogt, and U. Seibert. A stand alone complex for the production of water, food, electrical power and salts for the sustainable development of small communities in remote areas. Desalination, 183:13-22, 2005. doi:10.1016. C185

[2] R. A. Howard. Dynamic programming and Markov processes. The MIT Press, Cambridge, Mass., 1960. C186

[3] M. Kaleta, W. Ogryczak, E. Toczyłowski, and I. Zółtowska. On multiple criteria decision support for suppliers on the competitive electric power market. Annals of Operations Research, 121 (1-4):79-104, 2003. doi:10.1023/A:1023351118725. C185

[4] P. A. Koklas and S. A. Papathanassiou. Component sizing for an autonomous wind-driven desalination plant. Renewable Energy, 31 (13):2122-2139, 2006. doi:10.1016. C185

[5] C. Koroneos, A. Dompros, and G. Roumbas. Renewable energy driven desalination systems modelling. Journal of Cleaner Production, 15 (5):449-464, 2007. doi:10.1016/j.jclepro.2005.07.017. C185, C191 
[6] B. S. Richards and A. I. Schäfer. Photovoltaic-powered desalination system for remote Australian communities. Renewable Energy, 28 (13):2013-2022, 2003. doi:10.1016/S0960-1481(03)00081-8. C185 


\section{Author addresses}

1. R. B. Webby, School of Mathematical Sciences, University of Adelaide, Adelaide, Australia.

mailto:roger. webby@adelaide.edu.au

2. J. Boland, Centre for Industrial and Applied Mathematics, University of South Australia, Adelaide, Australia.

3. A. V. Metcalfe, School of Mathematical Sciences, University of Adelaide, Adelaide, Australia. 